\title{
A importância da formação docente ${ }^{12}$
}

Linda Darling-Hammond

Professora de Educação

na Universidade Stanford e diretora executiva da Comissão Nacional de Ensino e do Futuro dos Estados Unidos da América ldh@stanford.edu

\begin{abstract}
Resumo: A despeito das persistentes críticas à formação docente, o peso de evidências substanciais indica que professores mais preparados para ensinar são mais bemsucedidos e confiantes com os alunos do que aqueles que estudaram pouco ou quase nada para se tornarem professores. Dados recentes também indicam que as reformas na formação docente, que criaram programas mais estreitamente integrados com uma extensa preparação prática, entrelaçados com cursos sobre ensino e aprendizagem, produzem professores que são mais eficazes e que têm maior probabilidade de entrar na carreira e de permanecer nela. Uma contribuição importante da formação docente é que ela desenvolve as habilidades dos professores para examinar o ensino sob a perspectiva dos alunos, que trazem diferentes experiências e referências à sala de aula.
\end{abstract}

Palavras-chave: Formação de professores. Reforma educacional. Profissionalização docente.

Artigo publicado originalmente em Journal of Teacher Education (v. 51, n. 3, p. 166-173, maio/jun. 2000), com o título "How teacher education matters". O Journal é uma publicação da American Association of Colleges for Teacher Education (Associação Americana de Faculdades para Formação de Professores). Os direitos de publicação no artigo na Cadernos Cenpec foram adquiridos dos editores.

2 Tradução de Leda Beck e revisão técnica de Paula Louzano. 
Ao longo dos últimos dez anos, a insatisfação pública com as escolas passou a incluir a insatisfação com a formação dos professores. Faculdades de Educação foram criticadas de várias formas como ineficientes na preparação dos professores para seu trabalho, insensíveis a novas demandas e alheias à prática, constituindo verdadeiras barreiras ao recrutamento dos mais brilhantes alunos para a carreira docente. Em mais de 40 estados dos Estados Unidos, os formuladores de políticas públicas criaram rotas alternativas para a certificação de professores, com a intenção de oferecer diferentes caminhos para chegar ao ensino, além dos tradicionais programas de formação de professores em universidades de quatro anos de duração. Embora algumas dessas rotas sejam programas cuidadosamente estruturados para egressos do ensino médio, outras são pouco mais do que sistemas de recrutamento de emergência. A única proposta educacional do presidente Bush, logo depois de sua eleição, em 1988, foi o estímulo à certificação alternativa de professores. Em 1995, a grande iniciativa educacional de Newt Gingrich foi a eliminação das regras para a certificação de professores³. Em 1999, Chester Finn e a Fundação Thomas B. Fordham publicaram um manifesto contra os requisitos da formação de professores, que consideraram uma barreira à carreira docente.

Vozes insatisfeitas vieram também de dentro da profissão (GOODLAD, 1990; HOLMES GROUP, 1986). Essas vozes, porém, clamaram pela revisão da formação docente para fortalecer sua base de conhecimento, suas conexões tanto com a prática como com a teoria e sua capacidade de apoiar o desenvolvimento de um ensino mais robusto. Propostas nos extremos deste espectro contrastam enormemente entre si. Uma abordagem substituiria a preparação em universidade com a formação em serviço focada no ensino de maneira pragmática, enquanto a outra expandiria a formação profissional atual, preparando os professores para uma prática baseada em conhecimento, e lidando, ao mesmo tempo, com a reforma das escolas e do ensino. Qual desses caminhos é mais promissor? Quais são as implicações para a qualidade dos professores e, muito mais importante, para a educação das crianças?

Nota Técnica: Nos Estados Unidos, as regras e procedimentos para certificação de professores varia muito de estado para estado. Geralmente, uma autoridade estadual (por exemplo, o Conselho Estadual de Educação) é responsável pela supervisão da certificação de professores. Normalmente, o bacharelado é o requerimento mínimo, juntamente com cursos sobre métodos pedagógicos e experiências práticas. Até os anos 1980, uma pessoa não podia ser professor se não tivesse completado um ano ou mais de formação pedagógica. Nos últimos 20 anos, esse requisito tornou-se opcional devido à promoção da rota alternativa para certificação docente. 
Embora os debates sobre essas questões sejam principalmente ideológicos, há um corpo crescente de evidências sobre os resultados de diferentes abordagens para a formação e o recrutamento docentes. Esta pesquisa mostra que a extensão e a qualidade da formação docente influi na qualidade dos professores, talvez agora ainda mais do que antes. A expectativa de que as escolas ensinem um grupo de alunos muito mais diverso, em um nível muito mais alto, cria demandas muito maiores para os professores. Ensinar a resolver problemas, criar e aplicar o conhecimento requer professores com conhecimentos profundos e flexíveis do conteúdo, que saibam que representar ideias de forma poderosa pode significar um processo produtivo de aprendizado para alunos que começam o ano escolar com diferentes níveis e tipos de conhecimento prévios. Professores que sejam capazes de avaliar como e o que os alunos estão aprendendo, e adaptar sua instrução a diferentes práticas de ensino.

SERÁ QUE AS ESCOLAS DE EDUCAÇÃo AJUdAM OS PROFESSORES A APRENDER?

Mesmo que todos concordem que há habilidades e conhecimento desejáveis num professor, muita gente acredita que qualquer um pode ensinar ou, pelo menos, que saber um determinado conteúdo é suficiente para que alguém ensine bem. Outros acreditam que se aprende melhor a ensinar, se é que pode ser aprendido, por tentativa e erro na prática do trabalho. As evidências sugerem decididamente o contrário. A revisão de pesquisas realizadas nos últimos 30 anos concluiu que, mesmo com os defeitos do atual sistema de formação e certificação de professores, aqueles amplamente preparados e certificados obtêm, em geral, melhores resultados e são mais bem-sucedidos com os alunos do que os professores sem esse tipo de preparação (ASHTON; CROCKER, 1986; EVERTSON; HAWLEY; ZLOTNIK, 1985; GREENBERG, 1983; HABERMAN, 1984; OLSEN, 1985).

Em campos variando de matemática e ciência a educação vocacional, leitura, educação primária e educação infantil, os pesquisadores constataram que os professores que têm mais conhecimento do ensino e do aprendizado obtêm melhores resultados e são mais eficazes com os alunos, especialmente em tarefas que requerem pensamento de alta ordem e solução de problemas. (Para uma visão dessa literatura, ver Darling-Hammond, 1999b.) Curiosamente, embora o conhecimento da matéria seja frequentemente considerado um fator importante para um ensino eficaz, parece que sua relação com o desempenho 
no ensino é curvilínea; ou seja, esse conhecimento exerce um efeito positivo até certo limite e depois perde influência. Além disso, medidas de conhecimento pedagógico, inclusive conhecimento sobre a aprendizagem, métodos de ensino e currículo, com mais frequênca influenciam o desempenho no ensino e muitas vezes exercem efeitos ainda mais fortes do que o conhecimento do conteúdo da matéria (ASHTON; CROCKER, 1986; BEGLE; GEESLIN, 1972; BYRNE, 1983; EVERTSON; HAWLEY; ZLOTNICK, 1985; FERGUSON; WOMACK, 1993; GUYTON; FAROKHI, 1987; MONK, 1994; PERKES, 1967-1968). Parece lógico que a habilidade pedagógica interaja com o conhecimento da matéria para estimular ou minar o desempenho do professor. Como Byrne (1983) sugere,

\begin{abstract}
na medida em que o conhecimento de um professor ou uma professora proveja a base para sua eficiência, o conhecimento mais relevante será aquele relacionado com o assunto a ser ensinado e com as estratégias pedagógicas relevantes para ensiná-lo aos tipos específicos de alunos aos quais será ensinado (p.14).
\end{abstract}

Por outro lado, estudos com professores admitidos com uma preparação curta constatam que os novatos tendem a ser menos satisfeitos com sua formação e têm maior dificuldade para planejar o currículo, ensinar, gerenciar a sala de aula e diagnosticar as necessidades de aprendizagem dos alunos. São menos capazes de adaptar o ensino para promover o aprendizado e há menos probabilidade de que vejam isso como parte de seu trabalho, tendendo a culpar os alunos quando o ensino não é eficiente. Diretores e colegas avaliam pior as habilidades instrucionais desses professores e eles abandonam a profissão em índices acima da média. 0 mais importante é que seus alunos aprendem menos, especialmente em áreas como leitura, escrita e matemática, que são cruciais para o sucesso escolar mais tarde (DARLING-HAMMOND, 1999b).

Para ilustrar essas constatações, o estudo de Gomez e Grobe (1990) sobre o desempenho de candidatos à certificação alternativa (CA) em Dallas, que recebem algumas semanas de capacitação antes de assumir responsabilidades integrais de ensino, descobriu que eles tinham um desempenho muito menos uniforme do que o de professores treinados da forma tradicional que tinham notas equivalentes nos exames de conteúdo feitos pelo Estado. De duas a 16 vezes mais novatos de CA receberam a avaliação "fraco" em cada fator de ensino examinado e seus alunos mostraram ganhos de desempenho expressivamente menores em linguagem e escrita.

Talvez não seja surpreendente que os professores da rota alternativa vindos de programas de curta duração manifestem menos satisfação com sua preparação e menos compromisso com a carreira do que outros professores 
(DARLING-HAMMOND; HUDSON; KIRBY, 1989; LUTZ; HUTTON, 1989). Problemas resultantes da preparação inadequada encabeçaram a lista de reclamações dos $20 \%$ dos candidatos de CA de Los Angeles que abandonaram os programas de capacitação de verão em 1984 e 1985, assim como a de muitos que ficaram mas manifestaram insatisfação (WRIGHT; MCKIBBON; WALTON, 1987). A análise de Stoddart (1992) revela que 53\% desses recrutas abandonaram a carreira nos primeiros seis anos de funcionamento do programa. Entre os candidatos de CA em Dallas, só metade conseguiu "se graduar" com sucesso para se tornarem professores em tempo integral, depois de seu primeiro ano como estagiários. Só $40 \%$ deles disseram que planejavam permanecer no ensino, em contraste com $72 \%$ dos recrutas formados à maneira tradicional (LUTZ; HUTTON, 1989).

Até gente muito inteligente e entusiasmada com o ensino não acredita na sua capacidade de ser bem-sucedida sem preparação, especialmente se é designada para dar aulas para as crianças que mais precisam de um professor capaz. 0 mais famoso programa fundado com essa ideia é o Teach for America (TFA), criado para recrutar brilhantes alunos de universidades para lecionar em escolas públicas de áreas carentes enquanto estudam para outras carreiras e profissões que não a docente. Se fosse possível provar que professores nascem professores, não são formados como professores, esses alunos brilhantes e entusiasmados poderiam tê-lo feito. Mesmo assim, quatro avaliações diferentes constataram que o programa de formação de verão, com três a oito semanas de duração, não preparou adequadamente os candidatos (GRADY; COLLINS; GRADY, 1991; POPKEWITZ, 1995; ROTH, 1993; TEXAS EDUCATION AGENCY, 1993), a despeito da inteligência e do entusiasmo de muitos deles. Vários sabiam que seu sucesso - e o de seus alunos - tinha sido comprometido por sua falta de acesso ao conhecimento necessário para ensinar. O formando Schorr (1993), da Universidade Yale, foi um dos muitos a mencionar essa preocupação:

Talvez como muitos egressos do TFA, eu tinha sonhos de libertar meus alunos da mediocridade da escola pública e de lhes oferecer uma educação tão boa como a que eu mesmo recebera. Mas eu não estava preparado [...] Foi ruim para mim e foi pior para os alunos. Muitos dos meus [...] acabaram abandonando a escola [...] Não fui um professor bem-sucedido e a perda, para os alunos, foi grande e real (p.317-318).

Esse sentimento contribuiu para a alta taxa de evasão do programa. Embora muitos dos recrutas relatem que, inicialmente, entraram no programa com a intenção de explorar o magistério como carreira, muitos também indicaram que abandonaram a ideia, desencorajados porque se sentiam malsucedidos. As estatísticas do TFA mostram que, dos que iniciaram o programa em 1990, 
58\% tinham saído antes do terceiro ano, uma taxa de evasão em dois anos que é três vezes maior do que a média nacional para novos professores. A Secretaria de Educação do Estado de Maryland constatou que $62 \%$ dos membros do TFA que começaram a trabalhar em Baltimore em 1992 deixaram o programa em dois anos.

Além do alto índice de evasão, estudos sobre programas alternativos de curta duração também verificaram que mesmo o pouco treinamento pedagógico oferecido tende a se concentrar em habilidades genéricas de ensino, em vez de pedagogia específica da área de conhecimento; em técnicas singulares, em vez de um leque de práticas de ensino; e em conselhos específicos, imediatos, em vez de pesquisa ou teoria (BLISS, 1992; STODDART, 1992; ZUMWALT, 1990).

Geralmente, supõe-se que a falta de cursos tradicionais e de estágios nesses programas será compensada por monitoramento e supervisão intensivos nos meses iniciais de magistério. Ironicamente, porém, a maioria dos estudos constatou que os mentores prometidos raramente materializavam-se (DARLING-HAMMOND, 1992).

Infelizmente, os professores novatos menos preparados são designados desproporcionalmente para os alunos mais carentes, nas escolas de baixa renda e com grande incidência de minorias populacionais (Comissão Nacional do Ensino e do Futuro da América [NCTAF, na sigla em inglês], 1996). No agregado, isso pode fazer uma diferença substancial sobre o que as crianças aprendem. Recentes estudos multinível sobre o desempenho dos alunos nos níveis da escola e do distrito ${ }^{4}$ escolar constataram uma influência substancial da qualificação dos professores no aprendizado dos alunos. A análise de Ferguson (1991) dos distritos escolares do Texas constatou que a expertise dos professores - inclusive suas notas no exame de certificação que mede habilidades básicas e conhecimento de ensino, mestrados e experiência respondia por uma parcela maior da variação interdistrital no desempenho dos alunos em leitura e matemática do que o nível socioeconômico dos alunos. Os efeitos eram tão fortes e as variações na expertise dos professores tão grandes que, depois de controlar o fator nível socioeconômico, as grandes disparidades em desempenho entre alunos brancos e negros foram quase totalmente atribuídas às diferenças nas qualificações de seus professores. Essa constatação contradiz a percepção comum de que o desempenho escolar

4 Nota Técnica: Nos Estados Unidos, as escolas estão organizadas em distritos escolas, que no Brasil seriam equivalentes às secretarias ou departamentos de educação dos municípios e estados. 
dos alunos é largamente em função de sua origem social e que as variáveis escolares fazem pouca diferença nos resultados educacionais.

Um estudo texano mais recente (FULLER, 1999) verificou que os alunos em distritos com maior proporção de professores com formação em cursos de longa duração tinham probabilidade expressivamente maior de passar nos testes de desempenho do Estado do Texas, uma vez controlados os fatores de nível socioeconômico do aluno e da escola e de experiência do professor. Da mesma forma, um estudo da Carolina do Norte (STRAUSS; SAWYER, 1986) constatou que as notas médias dos professores nos Exames Nacionais de Ensino (NTE, na sigla em inglês), que medem conhecimento da matéria e de ensino, tiveram um grande efeito nos índices de aprovação dos alunos nos testes estaduais. Um aumento de $1 \%$ na qualidade do professor (medido pelas notas do NTE) foi associado com um declínio de 3\% a 5\% no porcentual de alunos reprovados no exame.

Um estudo recente, no nível da escola, sobre o desempenho em testes de matemática em escolas de ensino médio na Califórnia (FETLER, 1999) constatou um forte relação negativa entre a média das notas dos alunos e o porcentual de professores com certificados de emergência, depois de controlar o nível de vulnerabilidade social dos alunos. Outro estudo californiano constatou que, em todos os níveis de renda, o desempenho de leitura de alunos de primário está fortemente relacionado à proporção de professores formados e treinados em cursos de longa duração (Secretaria de Educação do Condado de Los Angeles, 1999), muito mais do que a proporção de professores iniciantes na escola. 0 estudo concluiu que "isso sustenta a constatação de que notas diferentes nos testes estão relacionadas à questão de formação de professores, não meramente devido à experiência dos professores em sala de aula".

\section{RESPOSTAS ÀS CRÍTICAS À FORMAÇÃO TRADICIONAL DOS PROFESSORES}

Antes que as faculdades de Educação fiquem cheias de si, há também razões para preocupação com os programas tradicionais de formação de professores. As críticas mais frequentes aos programas tradicionais de formação docente incluem a pressão do tempo num curso de quatro anos, que torna difícil aprender o suficiente tanto sobre a área de conhecimento específica como sobre pedagogia; a fragmentação dos cursos relacionados ao conteúdo a ser ensinado e aos de pedagogia; a divisão entre a formação universitária (teoria) e a formação na escola (prática); o conteúdo fraco de muitos cursos que não 
se baseiam em conhecimento sistematicamente desenvolvido; a falta de treinamento prático adequado; e a falta de recursos em muitos programas de formação que servem como "caça-níqueis" para suas universidades, o que perpetua quase tudo descrito acima.

Ao longo da última década do século XX, muitas faculdades de Educação e distritos escolares começaram a mudar essas condições. Estimuladas pelos esforços do Grupo Holmes e da Rede Nacional para Renovação Educacional, mais de 300 faculdades de Educação criaram programas que se estendem para além da tradicional licenciatura de quatro anos, permitindo, assim, um estudo mais extensivo das disciplinas a serem ensinadas, além de cursos de pedagogia mais integrados com a prática por meio de estágios intensivos nas escolas. Alguns são programas de pós-graduação de um ou dois anos, servindo alunos recém-formados em licenciatura ou professores de carreira. Outros são modelos de cinco anos de duração, que oferecem um programa extensivo de preparação para professores. Em ambos os casos, como o quinto ano permite que os alunos devotem suas energias exclusivamente para a tarefa de se preparar para ensinar, esses programas oferecem um ano de experiência clínica ${ }^{5}$ na escola e conectam essa experiência aos demais cursos de ensino e aprendizado.

Muitos desses programas de formação inicial uniram-se às secretarias de Educação locais para criar escolas de desenvolvimento profissional, onde a preparação clínica de noviços pode ser mais bem estruturada. Como os hospitais-escola na medicina, esses centros de formação visam a prover locais de prática no estado da arte e também são organizados para amparar a formação de novos profissionais, estender o desenvolvimento profissional de professores veteranos e promover a investigação e a pesquisa colaborativas. Essas abordagens assemelham-se a reformas da formação docente no exterior. Países como Alemanha, Bélgica, França e Luxemburgo há tempos requerem dos professores potenciais de dois a três anos de estudos de pós-graduação, além do bacharelado, e incluem um estágio intensamente supervisionado numa escola afiliada da universidade.

Vários estudos recentes constataram que os graduados por programas extensivos (em geral, programas de cinco anos de duração) estão não apenas mais satisfeitos com sua preparação, mas também são vistos por seus colegas,

5 Nota Técnica: Neste texto, e na concepção da autora, a expressão “clínica" se refere a um modelo de formação docente similar ao da área médica, em que o professor realiza estágio por meio de "residência pedagógica" em escolas parceiras das faculdades de Educação. 
diretores e professores como mais bem preparados, são tão eficazes com os alunos quanto professores muito mais experientes e têm probabilidade muito mais alta de entrar e permanecer na carreira do que seus pares formados por programas tradicionais de quatro anos de duração (ANDREW, 1990; ANDREW; SCHWAB, 1995; ARCH, 1989; DENTON; PETERS, 1988; DYAL, 1993; SHIN, 1994). De fato, as taxas de entrada e retenção desses programas são tão mais altas do que as dos programas de quatro anos - que, por sua vez, têm taxas muito mais altas do que as dos programas alternativos de curta duração - que fica muito mais barato preparar professores de carreira dessa forma, uma vez levados em consideração os custos de preparação, recrutamento, indução e substituição devida a abandono da carreira (DARLING-HAMMOMD, 1999a).

Geralmente, esses novos programas engajam professores potenciais no estudo de pesquisas e na condução de suas próprias investigações por meio de casos, pesquisa-ação e desenvolvimento de portfólios estruturados sobre a prática. Eles vislumbram o professor como alguém que aprende ao ensinar, em vez de alguém que já acabou de aprender a ensinar, e veem a tarefa de educar os professores como o desenvolvimento da capacidade de inquirir com sensibilidade e sistematicamente sobre a natureza do aprendizado e sobre os efeitos do ensino. Esta é uma abordagem da produção de conhecimento como a que Dewey (1929) buscava, visando a empoderar os professores com maior entendimento de situações complexas, em vez de controlá-los com fórmulas simplistas ou rotinas de ensino do tipo pré-moldado:

O domínio de métodos científicos e de conteúdo sistematizado liberta os indivíduos; capacita-os a ver novos problemas, divisar novos procedimentos e, em geral, produz mais diversidade do que uniformidade preestabelecida (p.12).

A noção de conhecimento para ensinar que Dewey propõe inclui investigação de problemas da prática como base para o julgamento profissional fundamentado tanto no conhecimento teórico como no prático. Se os professores investigam os efeitos de seu ensinar no aprendizado dos alunos, e se estudam o que outros aprenderam, acabam por entender que ensinar é uma ação intrinsecamente não rotineira. Tornam-se sensíveis à variação e mais conscientes sobre o que funciona para quais propósitos e em quais situações. 0 acesso ao conhecimento específico permite-lhes tomar decisões de maneira mais atenta.

A formação para a investigação também ajuda os professores a aprender como olhar para o mundo sob múltiplas perspectivas, inclusive as dos alunos, cujas experiências são muito diferentes da do professor, e a usar esse conhecimento 
para desenvolver pedagogias que podem atingir diferentes aprendizes. Aprender a alcançar os alunos, tanto os fáceis como os difíceis de conhecer, requer atravessar fronteiras, a habilidade de extrair conhecimento de outrem e de entendê-lo quando ele é oferecido. Como observou Delpit (1995), "todos nós interpretamos comportamentos, informação e situações por meio de nossas próprias lentes culturais; essas lentes funcionam involuntariamente, abaixo do nível da consciência, fazendo parecer que nossa própria visão é simplesmente 'o jeito que é’” (p. 151). Bons professores devem desenvolver uma consciência de suas perspectivas e como elas podem ser ampliadas, para evitar um "viés comunicêntrico" (GORDON, 1990), que limita seu entendimento dos alunos.

Talvez este seja o papel mais importante das universidades na preparação dos professores: desenvolver a habilidade de ver para além da própria perspectiva, colocar-se no lugar do aluno e entender o significado da experiência em termos de aprendizado. Um dos grandes defeitos do "mito da pessoa brilhante" no ensino é que ele presume que qualquer um pode ensinar o que sabe para qualquer outra pessoa. No entanto, gente que nunca estudou ensino e aprendizagem muitas vezes tem enorme dificuldade para entender como ensinar algo que eles mesmos aprenderam sem esforço e quase subconscientemente. Quando os outros não aprendem só por ouvir contar, o professor intuitivo muitas vezes fica frustrado e impotente para prosseguir. Com frequência, isso leva a se ressentir dos alunos por não validarem os esforços do professor não treinado. Além disso, indivíduos que não sofreram uma boa intervenção durante a sua formação como professor geralmente mantêm uma única perspectiva cultural e cognitiva, o que torna difícil para eles entender as experiências, percepções e bases de conhecimento que influenciam profundamente as abordagens ao aprendizado por alunos que são muito diferentes do próprio professor. $A$ capacidade para entender o outro não é inata; ela é desenvolvida por meio de estudo, reflexão, experiência guiada e investigação.

Entre as ferramentas cada vez mais usadas para isso pelos formadores de professores estão as pesquisas que engajam os futuros professores na investigação do aprendizado e da vida dos alunos, e na análise dos diferentes resultados do ensino. Essas pesquisas incluem estudos de caso de crianças, realizados pelos futuros professores, ao mesmo tempo que estudam desenvolvimento e aprendizagem, chegando, assim, a entender melhor as experiências e o pensamento das crianças; estudos de comunidades, investigando bairros de forma a iluminar as experiências de vida, costumes e cultura de diferentes grupos de pessoas; pesquisas sobre o aprendizado dos alunos, como o trabalho desenvolvido para o processo de certificação do 
Conselho Nacional de Padrões da Profissão Docente ${ }^{6}$, em que o professor avalia o processo de aprendizagem de três alunos diferentes ao longo do tempo ou monta portfólios que usam trabalhos relacionados ao ensino e à aprendizagem para analisar os efeitos de sua prática docente; e investigações baseadas em problemas de prática, que são entendidas mediante pesquisa-ação combinada com revisão de outras pesquisas. Essas ferramentas permitem a aplicação de princípios teóricos aos problemas em contextos específicos e, ao mesmo tempo, apropriadamente, complicam os esforços para extrair generalizações da prática. Um ainda pequeno, mas crescente, corpo de pesquisa sugere que tais estratégias podem ajudar os professores a entender mais profundamente as muitas variáveis que influenciam seu trabalho. Por exemplo: nos estudos de caso e portfólios que requerem que os professores examinem o aprendizado dos alunos em relação ao seu ensino, os professores afirmam que esse tipo de análise acaba por enriquecer a habilidade deles para entender os efeitos de suas ações e os ajuda a responder melhor às necessidades de diferentes alunos. (Para um compêndio dessa literatura, ver Darling-Hammond e Snyder, 2000.) Uma das maneiras em que isso ocorre é pelo processo de tentar ver as práticas de ensino e da sala de aula sob as perspectivas dos alunos que as vivem. À medida que os professores olham para além de suas próprias ações e avaliam-nas à luz do conhecimento adquirido por eles mesmos sobre alunos individuais e do conhecimento profissional sobre os fatores que influenciam o desenvolvimento e o aprendizado, tornam-se mais sábios sobre as muitas maneiras em que ensino e aprendizagem interagem.

Um compromisso com a pesquisa, o alargamento das perspectivas e 0 cruzamento de fronteiras são características fundamentais do ideal da educação universitária. Com efeito, a base das primeiras universidades era que elas tentavam reunir pesquisadores de todo o mundo. Elas buscavam criar maneiras de compartilhar perspectivas de várias áreas geográficas, culturas e disciplinas, como base para desenvolver conhecimento e encontrar a verdade. Se as universidades vão continuar a fazer a importante contribuição que podem fazer para a educação dos professores, precisam perseguir esses ideais de construção do conhecimento e busca da verdade por meio da criação de uma

6 Nota Técnica: 0 Conselho Nacional busca elevar o status, a voz e o papel de professores na definição de uma verdadeira profissão. Isso inclui: 1) conscientizar o público sobre a complexidade cognitiva do trabalho dos professores e sua natureza colaborativa e especializada; 2) estabelecer altos padrões para o ingresso e o crescimento na profissão; e 3) reconhecer o ensino de qualidade por meio de um rigoroso processo de certificação profissional, comparável aos de outras grandes profissões, como medicina, engenharia e direito. 
práxis genuína entre ideias e experiências, honrando a prática em conjunto com a reflexão e a pesquisa, e ajudando os professores a ir além de suas fronteiras pessoais e apreciar as perspectivas daqueles a quem vão ensinar.

\section{DISCLAIMER}

While every effort has been made to ensure that the contents of this publication are factually correct, neither the authors nor the publisher accepts, and they hereby expressly exclude to the fullest extent permissible under applicable law, any and all liability arising from the contents published in this article, including, without limitation, from any errors, omissions, inaccuracies in original or following translation, or for any consequences arising therefrom. Nothing in this notice shall exclude liability which may not be excluded by law.

\section{RENÚNCIA DE RESPONSABILIDADE}

Embora tenham sido feitos todos os esforços para assegurar que o conteúdo desta publicação seja factualmente correto, nem os autores nem o editor aceitam, e por meio desta nota expressamente excluem, da forma mais completa possível sob a lei vigente, qualquer e toda responsabilidade decorrente do conteúdo publicado neste artigo, incluindo, mas sem se limitar a ela, a responsabilidade decorrente de erros, omissões, imprecisões no original ou na tradução que o acompanha, ou de quaisquer consequências disso. Nada nesta nota exclui a responsabilidade que não pode ser excluída por lei. 


\section{How teacher education matters}

Abstract: Despite longstanding criticisms of teacher education, the weight of substantial evidence indicates that teachers who have had more preparation for teaching are more confident and successful with students than those who have had little or none. Recent evidence also indicates that reforms of teacher education creating more tightly integrated programs with extended clinical preparation interwoven with coursework on learning and teaching produce teachers who are both more effective and more likely to enter and stay in teaching. An important contribution of teacher education is its development of teachers' abilities to examine teaching from the perspective of learners who bring diverse experiences and frames of reference to the classroom.

Keywords: Teacher education. Educational reform. Teacher professionalization. 
ANDREW, M. The differences between graduates of four-year teacher preparation programs. Journal of Teacher Education, v. 41, p. 45-51, 1990.

; SCHWAB, R. L. Has reform in teacher education influenced teacher performance? An outcome assessment of graduates of eleven teacher education programs. Action in Teacher Education, v. 17, p. 43-53, 1995.

$\mathrm{ARCH}$, E. C. Comparison of student attainment of teaching competence in traditional preservice and fifth-year Master of Arts in teaching programs. Estudo apresentado no encontro anual da American Educational Research Association, San Francisco, Califórnia, abr. 1989.

ASHTON, P.; CROCKER, L. Does teacher certification make a difference? Florida Journal of Teacher Education, v. 3, p. 73-83, 1986.

BEGLE, E. G.; GEESLIN, W. Teacher effectiveness in mathematics instruction. (National Longitudinal Study of Mathematical Abilities Reports $n^{\circ}$ 28.) Washington, DC: Mathematical Association of America e National Council of Teachers of Mathematics, 1972.

BLISS, T. Alternate certification in Connecticut: reshaping the profession. Peabody Journal of Education, v. 67, n. 3, p. 35-54, 1992.

BYRNE, C. Teacher knowledge and teacher effectiveness: a literature review, theoretical analysis, and discussion of research strategy. Estudo apresentado no encontro da Northeastern Educational Research Association, Ellenville, NY, 1983.

DARLING-HAMMOND, L. Teaching and knowledge: policy issues posed by alternative certification for teachers. Peabody Journal of Education, v. 67, n. 3, p. 123-154, 1992.

. Solving the dilemmas of teacher supply, demand, and standards: how we can ensure a competent, caring, and qualified teacher for every child. Nova York: National Commission on Teaching and America's Future, 1999a.

Teaching quality and student achievement: a review of state policy evidence. Seattle, WA: Center for the Study of Teaching and Policy, Universidade de Washington, 1999b.

; HUDSON, L.; KIRBY, S. Redesigning teacher education: opening the door for new recruits to science and mathematics teaching. Santa Monica, CA: Rand, 1989. 
SNYDER, J. Authentic assessment of teaching in context. Journal of Teaching and Teacher Education, v. 16, p. 523-545, 2000.

DENTON, J. J.; PETERS, W. H. Program assessment report: curriculum evaluation of a non-traditional program for certifying teachers. College Station, Texas: Universidade A \& M do Texas, 1988.

DEWEY, J. The sources of a science of education. Nova York: Horace Liveright, 1929.

DYAL, A. B. An exploratory study to determine principals' perceptions concerning the effectiveness of a fifth-year preparation program. Estudo apresentado no encontro anual da Mid-South Educational Research Association, New Orleans, Louisiana, 1993.

EVERTSON, C.; HAWLEY, W.; ZLOTNICK, M. Making a difference in educational quality through teacher education. Journal of Teacher Education, v. 36, n. 3, p. 2-12, 1985.

FERGUSON, R. F. Paying for public education: new evidence on how and why money matters. Harvard Journal on Legislation, v. 28, n. 2, p. 465-498, 1991.

FERGUSON, P.; WOMACK, S. T. The impact of subject matter and education coursework on teaching performance. Journal of Teacher Education, v. 44, n. 1, p. 55-63, 1993.

FETLER, M. High school staff characteristics and mathematics test results. Education Policy Analysis Archives, v. 7, 24 mar. 1999. Disponível em: <http:// epaa.asu.edu>.

FULLER, E. J. Does teacher certification matter? A comparison of TAAS performance in 1997 between schools with low and high percentages of certified teachers. Austin: Charles A. Dana Center, Universidade do Texas em Austin, 1999.

GOMEZ, D. L.; GROBE, R. P. Three years of alternative certification in Dallas: Where are we? Estudo apresentado no encontro anual da American Educational Research Association, Boston, abr. 1990.

GOODLAD, J. Teachers for our nation's schools. San Francisco: Jossey-Bass, 1990.

GORDON, E. W. Coping with communicentric bias in knowledge production in the social sciences. Educational Researcher, v. 19, n. 3, p. 14-19, 1990.

GRADY, M. P.; COLLINS, P.; GRADY, E. L. Teach for America 1991 Summer Institute evaluation report. Educational Leadership, v. 43, n. 3, p. 4-10, 1991. 
GREENBERG, J. D. The case for teacher education: Open and shut. Journal of Teacher Education, v. 34, n. 4, p. 2-5, 1983.

GUYTON, E.; FAROKHI, E. Relationships among academic performance, basic skills, subject matter knowledge and teaching skills of teacher education graduates. Journal of Teacher Education, v. 38, n. 5, p. 37-42, set./out. 1987.

HABERMAN, M. An evaluation of the rationale for required teacher education: Beginning teachers with or without teacher preparation. Estudo preparado para a National Commission on Excellence in Teacher Education, Universidade de Wisconsin-Milwaukee, set. 1984.

HOLMES GROUP. Tomorrow's teachers: a report of the Holmes Group. East Lansing, Michigan, 1986.

LOS Angeles County Office Of Education, 1999.

LUTZ, F. W.; HUTTON, J. B. Alternative teacher certification: its policy implications for classroom and personnel practice. Educational Evaluation and Policy Analysis, v. 11, n. 3, p. 237-254, 1989.

MONK, D. H. Subject matter preparation of secondary mathematics and science teachers and student achievement. Economics of Education Review, v. 13, n. 2, p. 125-145, 1994.

National Commission on Teaching and America's Future. What matters most: teaching for America's future. New York, 1996.

OLSEN, D. G. The quality of prospective teachers: education vs. noneducation graduates. Journal of Teacher Education, v. 36, n. 5, p. 56-59, 1985.

PERKES, V. A. Junior high school science teacher preparation, teaching behavior, and student achievement. Journal of Research in Science Teaching, v. 6, n. 4, p. 121-126, 1967-1968.

POPKEWITZ, T. S. Policy, knowledge, and power: some issues for the study of educational reform. In: COOKSON, P.; B. SCHNEIDER, B. (Eds.). Transforming schools: Trends, dilemmas and prospects. New York: Garland Press, 1995.

ROTH, R. A. Teach for America 1993 Summer Institute: Program Review, 1993.

SCHORR, J. Class action: what Clinton's National Service Program could learn from “Teach for America”. Phi Delta Kappan, v. 75, n. 4, p. 315-318, dez. 1993.

SHIN, H.-S. Estimating future teacher supply: an application of survival analysis. Estudo apresentado no encontro anual da American Educational Research Association, New Orleans, Louisiana, 1994. 
STODDART, T. An alternate route to teacher certification: preliminary findings from the Los Angeles Unified School District Intern Program. Peabody Journal of Education, v. 67, n. 3, 1992.

STRAUSS, R. P.; SAWYER, E. A. Some new evidence on teacher and student competencies. Economics of Education Review, v. 5, n. 1, p. 41-48, 1986.

TEXAS EDUCATION AGENCY. Teach for America visiting team report. Meeting minutes of Texas State Board of Education Meeting (Appendix B), Austin, 1993.

WRIGHT, D. P.; MCKIBBON, M.; WALTON, P. The effectiveness of the teacher trainee program: an alternate route into teaching in California. Sacramento: California Commission on Teacher Credentialing, 1987.

ZUMWALT, K. Alternate routes to teaching: three alternative approaches. New York: Teachers College, Universidade Columbia, 1990. 
